\title{
Is Intra-Arterial Treatment for Acute Ischemic Stroke Less Effective in Women than in Men?
}

\author{
Inger R. de Ridder ${ }^{a} \quad$ Puck S.S. Fransen ${ }^{a} \quad$ Debbie Beumer ${ }^{b}$ \\ Olvert A. Berkhemer ${ }^{a, c}$ Lucie A. van den Berg ${ }^{c}$ Marieke J. Wermer ${ }^{d}$ \\ Hester Lingsma ${ }^{a}$ Wim H. van Zwam ${ }^{b}$ Yvo B. Roos ${ }^{c}$ \\ Robert J. van Oostenbrugge $^{b} \quad$ Charles B. Majoie ${ }^{c} \quad$ Aad van der Lugt $^{a}$ \\ Diederik W.J. Dippela for the MR CLEAN Investigators \\ a Erasmus MC University Medical Center, Rotterdam, b Maastricht University Medical Center, \\ Maastricht, ' Academic Medical Center, Amsterdam, and d Leiden University Medical Center, \\ Leiden, The Netherlands
}

\section{Key Words}

Cerebral infarction $\cdot$ Endovascular treatment $\cdot$ Sex $\cdot$ Outcome

\begin{abstract}
Introduction: Stroke etiology and outcome after ischemic stroke differ between men and women. We examined if sex modifies the effect of intra-arterial treatment (IAT) in a randomized clinical trial of IAT for acute ischemic stroke in the Netherlands (MR CLEAN). Patients and Methods: The primary outcome was the score on the modified Rankin scale at 90 days. We tested for interaction between sex and treatment and estimated the treatment effect by sex with multiple ordinal logistic regression with adjustment for prognostic factors. Results: All 500 patients were included in the analysis; 292 (58.4\%) were men. The treatment effect (adjusted common odds ratio) was 2.39 [95\% confidence interval (CI) 1.55-3.68] in men and 0.99 $\left(95 \%\right.$ CI 0.60-1.66) in women ( $\left.p_{\text {interaction }}=0.016\right)$. In women, mortality was higher in the intervention group than in the control group ( 24 vs. $15 \%, p=0.07$ ). Serious adverse events occurred more often in women than in men undergoing intervention. There were no differences in neuro-imaging outcomes. Discussion and Conclusion: Contrary to other studies, we found a significant interaction between sex and treatment effect in the MR CLEAN trial. Pooled analyses of all published thrombectomy trials did not confirm this finding. In MR CLEAN, women seem to have a slightly more unfavorable profile, causing higher mortality and more serious adverse events, but insufficient to explain the absence of an overall effect. This suggests a play of chance and makes it clear that IAT should not be withheld in women.

(C) 2016 The Author(s)

Published by S. Karger AG, Basel
\end{abstract}

I.R.d.R. and P.S.S.F. contributed equally to this work. The MR CLEAN trial is registered under No. NTR1804 in the Dutch trial register and under ISRCTN10888758 in the ISRCTN register. 
de Ridder et al.: Is Intra-Arterial Treatment for Acute Ischemic Stroke Less Effective in Women than in Men?

\section{Introduction}

Women in high-income countries have a higher lifetime stroke risk and higher stroke mortality than men. Poor outcome after stroke is more frequent in women, which may add to the anticipated increase in burden of stroke [1]. Female stroke survivors are more likely to be disabled and single after stroke and are institutionalized 3.5 times more often than male survivors [2]. The sex-specific effects on stroke incidence and outcome might be contributed to female physiology, which differs from men in terms of coagulation, immunity and hormone exposure [2]. Recently, intra-arterial treatment (IAT) with stent thrombectomy devices for acute ischemic stroke (AIS) has been proven safe and effective [3-7]. In this study, we aim to assess if sex modifies the effect of IAT in AIS.

\section{Methods}

Detailed methods of the MR CLEAN trial have been described earlier $[3,8]$. In short, MR CLEAN was a multicenter randomized-controlled trial of IAT for AIS. Patients were randomized to IAT plus usual care, or usual care alone. Eligible patients had a proximal arterial occlusion in the anterior cerebral circulation that was confirmed on vessel imaging and could be treated within $6 \mathrm{~h}$ after symptom onset. Approval was obtained from a central medical ethics committee and the research board of each participating center, and all participants (or legal representatives) provided written informed consent.

Outcome

The primary outcome measure was the score on the modified Rankin scale (mRS) at 90 days. Secondary outcome measures included 90-day functional independence (mRS 0-2) and mortality. Radiological outcome measures included arterial recanalization measured with the modified thrombolysis in cerebral infarction (mTICI) score on digital subtraction angiography and final infarct volume on non-contrast CT at 5-7 days [9]. Safety parameters included hemorrhagic complications, progression of ischemic stroke, recurrent ischemic stroke and death. Symptomatic intracranial hemorrhage was defined as neurological deterioration of 4 or more points on the National Institute of Health Stroke Scale (NIHSS) with confirmed intracranial hemorrhage on neuroimaging.

\section{Statistical Analysis}

The primary effect parameter was the adjusted common odds ratio (acOR) for a shift in the direction of better outcome on the mRS, which was estimated with multivariable ordinal logistic regression. We looked for the interaction between sex and treatment effect by adding a multiplicative term in the multivariable ordinal logistic regression model. The acOR and all secondary effect variables were adjusted for pre-specified potential imbalances in major prognostic variables between the intervention and control groups: age, NIHSS at baseline, time from onset to randomization, previous stroke, atrial fibrillation, diabetes mellitus and internal carotid artery terminus occlusion, and were reported with $95 \%$ confidence intervals (CI). All statistical analyses were performed with Stata/SE 13.1 (StataCorp, College Station, Tex., USA).

\section{Results}

All 500 patients were included in the analysis; 292 (58.4\%) were men. Women were on average 4 years older than men and had a lower percentage mRS 0 before randomization and slightly more atrial fibrillation (table 1 ). The overall effect of the intervention on shift on the mRS at 90 days was acOR 1.67 (95\% CI; 1.21-2.30). The acOR was 0.99 (95\% CI; 0.60-1.66) in women and 2.39 (95\% CI; 1.55-3.68) in men (fig. 1). The p value for interaction was 0.016 . In men, mortality in the intervention group was lower than in the control group (15 vs. $21 \%$, $\mathrm{p}=0.17$ ), whereas in women mortality was higher in the intervention group than in the control group ( 24 vs. $15 \%, p=0.07$ ). Functional independence at 90 days was reached in $36 \%$ 


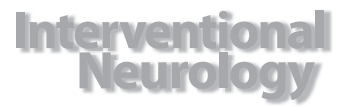

Table 1. Baseline clinical characteristics by sex

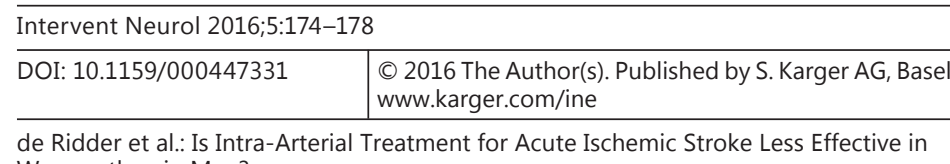

de Ridder et al.: Is Intra-Arterial Treatment for Acute Ischemic Stroke Less Effective in Women than in Men?

\begin{tabular}{lcc}
\hline & $\begin{array}{l}\text { Women } \\
(\mathrm{n}=208)\end{array}$ & $\begin{array}{l}\text { Men } \\
(\mathrm{n}=292)\end{array}$ \\
\hline Age, years & $68(55-78)$ & $64(56-74)$ \\
Pre-stroke mRS 0 & $157(75)$ & $247(85)$ \\
Pre-stroke mRS 1--2 & $38(18)$ & $37(13)$ \\
Atrial fibrillation & $60(29)$ & $75(26)$ \\
NIHSS & $17(14-21)$ & $18(15-22)$ \\
ICA-T occlusion & $50(24)$ & $84(29)$ \\
Time from onset to & $197(149-258)$ & $204(153-262)$ \\
\hline
\end{tabular}

Values are expressed as median (interquartile range) or $\mathrm{n}(\%)$. ICA-T = Internal carotid artery terminus.

Table 2. Safety outcomes by sex and treatment allocation

\begin{tabular}{|c|c|c|c|c|c|c|}
\hline \multirow[t]{2}{*}{ Treatment allocation } & \multicolumn{3}{|l|}{ Women } & \multicolumn{3}{|l|}{ Men } \\
\hline & $\begin{array}{l}\text { IAT } \\
(n=98)\end{array}$ & $\begin{array}{l}\text { Control } \\
(n=110)\end{array}$ & $\begin{array}{l}\mathrm{p} \\
\text { value }\end{array}$ & $\begin{array}{l}\text { IAT } \\
(n=135)\end{array}$ & $\begin{array}{l}\text { Control } \\
(n=157)\end{array}$ & $\begin{array}{l}\mathrm{p} \\
\text { value }\end{array}$ \\
\hline Death within 7 days & $16(16)$ & $6(5)$ & 0.01 & $17(13)$ & $21(13)$ & 0.84 \\
\hline Death within 30 days & $24(24)$ & $16(15)$ & 0.07 & $20(15)$ & $33(21)$ & 0.17 \\
\hline Any SAE & $45(46)$ & $40(36)$ & 0.16 & $65(48)$ & $73(47)$ & 0.78 \\
\hline Hemicraniectomy & $5(5)$ & $3(3)$ & 0.37 & $9(7)$ & $6(6)$ & 0.92 \\
\hline sICH & $9(9)$ & $4(4)$ & 0.10 & $9(7)$ & $13(8)$ & 0.60 \\
\hline Recurrent stroke & $6(6)$ & $1(1)$ & 0.04 & $7(5)$ & $0(0)$ & 0.00 \\
\hline Progression of stroke & $20(20)$ & $18(16)$ & 0.45 & $26(19)$ & 29 (19) & 0.64 \\
\hline
\end{tabular}

Values are expressed as n (\%). SAE = Serious adverse event; sICH = symptomatic intracerebral hemorrhage.

of the men and $29 \%$ of the women [aOR in men 2.19 (95\% CI; 1.24-3.87) and in women 2.05 (95\% CI; 0.96-4.35)]. For women, this results in violation of the proportional odds assumption and a neutral common odds ratio. There were no differences in revascularization (mTICI score) between women and men (both 59\% mTICI 2B or 3). In men, the difference in infarct volume was statistically significant $(27 \mathrm{ml}, \mathrm{p}=0.01)$, whereas in women this was not $(12 \mathrm{ml}$, $\mathrm{p}=0.34)$. Women in the intervention group experienced more serious adverse events, including recurrent stroke, than controls ( 46 vs. $36 \%, p=0.16$; table 2).

\section{Discussion}

We studied the interaction between sex and IAT and showed that men experienced a major benefit from IAT in MR CLEAN, whereas we did not find a beneficial treatment effect in women. In women, the proportionality assumption required for ordinal analysis was not met (online suppl. table; see www.karger.com/doi/10.1159/000447331 for all online suppl. material), and the effect on reaching independence was offset by an increase in mortality and severe disability. Moreover, women had a more unfavorable risk profile with a lower percentage mRS 0 before randomization and slightly more atrial fibrillation, and they expe- 

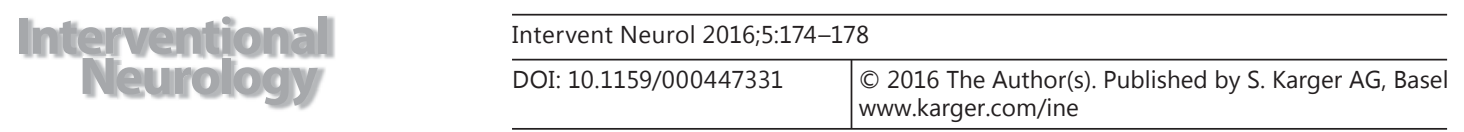

de Ridder et al.: Is Intra-Arterial Treatment for Acute Ischemic Stroke Less Effective in Women than in Men?

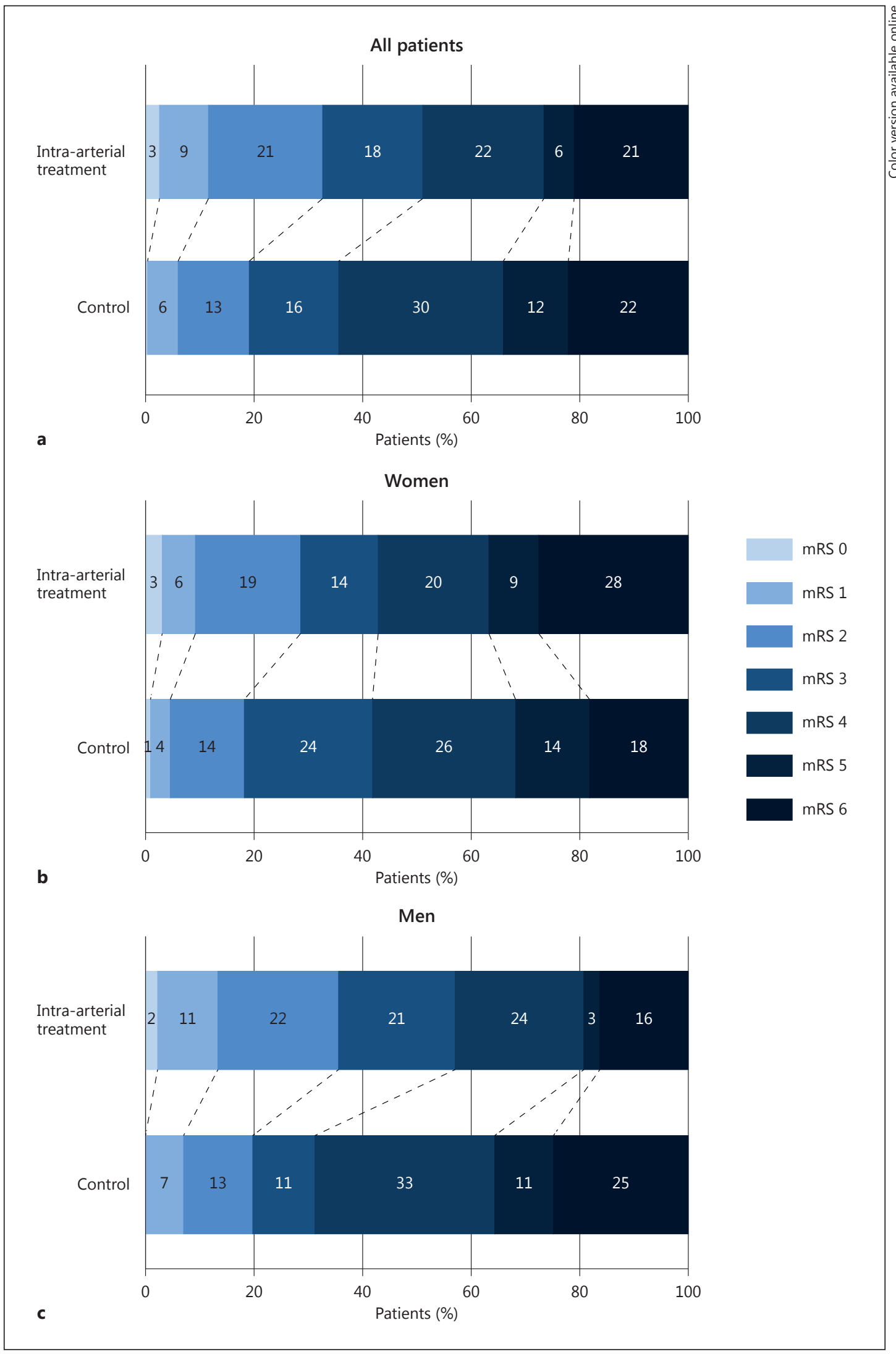

Fig. 1. Effects of the intervention on the primary outcome in all patients (a), women (b) and men (c). 
de Ridder et al.: Is Intra-Arterial Treatment for Acute Ischemic Stroke Less Effective in Women than in Men?

rienced more serious adverse events in the intervention group compared to men. However, this does not totally explain the large difference in the treatment effect, which suggests a play of chance.

Several studies have assessed sex differences in outcome recanalization and functional outcome after IAT for AIS [7, 10-12]. However, these studies did not report baseline characteristics by sex. Therefore, we could not compare the baseline characteristics of women in our study to the baseline profile of women in other studies. None of these studies found differences in the recanalization rate and favorable outcome between women and men. Pooled analyses of all published thrombectomy trials also showed no difference between men and women in a favorable outcome after IAT [13]. The increased mortality and poor outcome among women compared to men in the MR CLEAN trial has no firm clinical or biological basis. These considerations suggest that a play of chance is the plausible explanation for our findings and make it clear that IAT should not be withheld in women.

\section{Disclosure Statement}

The authors have no conflicts of interest to disclose.

\section{References}

1 Sex differences and stroke prevention (editorial). Lancet Neurol 2014;13:339.

2 Howe MD, McCullough LD: Prevention and management of stroke in women. Expert Rev Cardiovasc Ther 2015;13:403-415.

3 Berkhemer OA, Fransen PS, Beumer D, et al: A randomized trial of intraarterial treatment for acute ischemic stroke. N Engl J Med 2015;372:11-20.

4 Campbell BC, Mitchell PJ, Kleinig TJ, et al: Endovascular therapy for ischemic stroke with perfusion-imaging selection. N Engl J Med 2015;372:1009-1018.

5 Goyal M, Demchuk AM, Menon BK, et al: Randomized assessment of rapid endovascular treatment of ischemic stroke. N Engl J Med 2015;372:1019-1030.

6 Jovin TG, Chamorro A, Cobo E, et al: Thrombectomy within $8 \mathrm{~h}$ after symptom onset in ischemic stroke. $\mathrm{N}$ Engl J Med 2015;372:2296-2306.

7 Saver JL, Goyal M, Bonafe A, et al: Stent-retriever thrombectomy after intravenous t-PA vs. t-PA alone in stroke. N Engl J Med 2015;372:2285-2295.

8 Fransen PS, Beumer D, Berkhemer OA, et al: MR CLEAN, a multicenter randomized clinical trial of endovascular treatment for acute ischemic stroke in the Netherlands: study protocol for a randomized controlled trial. Trials 2014;15:343.

9 Zaidat OO, Yoo AJ, Khatri P, et al: Recommendations on angiographic revascularization grading standards for acute ischemic stroke: a consensus statement. Stroke 2013;44:2650-2663.

10 Goyal M, Demchuk AM, Menon BK, et al: Randomized assessment of rapid endovascular treatment of ischemic stroke. N Engl J Med 2015;372:1019-1030.

11 Lutsep HL, Hill MD: Effects of sex on mechanical embolectomy outcome. J Stroke Cerebrovasc Dis 2012;21: 240-242.

12 Arnold M, Kappeler L, Nedeltchev K, et al: Recanalization and outcome after intra-arterial thrombolysis in middle cerebral artery and internal carotid artery occlusion: does sex matter? Stroke 2007;38:1281-1285.

13 Goyal M, Menon BK, van Zwam WH, et al: Endovascular thrombectomy after large-vessel ischaemic stroke: a meta-analysis of individual patient data from five randomised trials. Lancet 2016;387:1723-1731. 